\title{
Field Emission from Self-Assembled Arrays of Lanthanum Monosulfide Nanoprotrusions
}

\author{
V. Semet, ${ }^{1}$ Vu Thien Binh, ${ }^{1}$ M. Cahay, ${ }^{2}$ K. Garre, ${ }^{2}$ S. Fairchild, ${ }^{3}$ L. Grazulis, ${ }^{3}$ J. W. Fraser, ${ }^{4}$ \\ D. J. Lockwood, ${ }^{4}$ S. Pramanik, ${ }^{5}$ B. Kanchibotla, ${ }^{5}$ and S. Bandyopadhyay ${ }^{5}$ \\ ${ }^{1}$ Equipe Emission Electronique, LPMCN-CNRS, Universite Claude Bernard Lyon 1, 69622 Villeurbanne, France \\ ${ }^{2}$ Department of Electrical and Computer Engineering and Computer Science, University of Cincinnati, Cincinnati, OH 45221, USA \\ ${ }^{3}$ Materials and Manufacturing Directorate, Air Force Research Laboratory, Wright-Patterson AFB, OH 45433, USA \\ ${ }^{4}$ Institute for Microstructural Sciences, National Research Council, Ottawa, ON, Canada K1A OR6 \\ ${ }^{5}$ Department of Electrical and Computer Engineering, Virginia Commonwealth University, Richmond, VA 23284, USA
}

Correspondence should be addressed to M. Cahay, marc.cahay@uc.edu

Received 30 October 2007; Accepted 10 January 2008

Recommended by Donglu Shi

\begin{abstract}
The field emission properties of LaS nanoprotrusions called nanodomes, formed by pulsed laser deposition on porous anodic alumina films, have been analyzed with scanning anode field emission microscopy. The voltage necessary to produce a given field emission current is $\sim 3.5$ times less for nanodomes than for thin films. Assuming the same work function for LaS thin films and nanoprotrusions, that is, $\sim 1 \mathrm{eV}$, a field enhancement factor of $\sim 5.8$ is extracted for the nanodome emitters from FowlerNordheim plots of the field emission data. This correlates well with the aspect ratio of the tallest nanodomes observed in atomic force micrograph measurements.
\end{abstract}

Copyright (c) 2008 V. Semet et al. This is an open access article distributed under the Creative Commons Attribution License, which permits unrestricted use, distribution, and reproduction in any medium, provided the original work is properly cited.

The promise of producing extremely high current densities in near-vacuum with no external source (such as heater, primary electron-beam, or intense light source) has generated significant interest in robust, reproducible, and fieldemission-based cold cathodes $[1,2]$. Several promising approaches to achieving cold cathode emission from a dense array of nanoscale field emitters have been investigated including metallic field emitter arrays (FEAs) or Spindt emitters [3], carbon nanotube (CNT) arrays [4], and arrays of semiconductor nanowires [5-9]. To date, metallic FEAs with uniformly high emission have been difficult to fabricate. They are prone to failure and present long-term reliability problems [3]. Carbon nanotubes (CNTs), on the other hand, can be fabricated easily, but the emission current density that they can support, while adequate for some applications, is currently not enough for mainstream high power devices such as traveling wave tubes (TWTs). Semiconductor and semimetallic nanowires therefore appear as perhaps the most promising route to realizing field emitters that can support a large total emission current, in particular if regular high density arrays of them can be fabricated in a self-assembled growth mode. Moreover, to achieve high current densities under low applied voltage, the field emitter material must have low work function. Rare earth monosulfides are therefore ideal for this purpose.

Recently, we reported the first successful deposition of lanthanum monosulfide (LaS) thin films on Si substrates using pulsed laser deposition (PLD) [10, 11]. They were flat layers composed of nanocrystallites having dimensions of the order of $5 \mathrm{~nm}$. The scanning anode field emission microscopy (SAFEM) technique was used to measure the field emission (FE) properties of these LaS thin films (about $0.1 \mu \mathrm{m}$ thick) [12]. It was shown experimentally that electrons are easily extracted by FE from patchwork areas of few nanometer dimensions having a measured effective work function of $\sim 1 \mathrm{eV}$, corresponding to some outcropping LaS nanocrystallites with (100) orientation, embedded in a $\sim 2.8 \mathrm{eV}$ work function surface related to other crystallographic orientations. A LaS thin film obtained by PLD can therefore be an excellent field emitter material, especially if we can take advantage of a field enhancement factor at the tip of nanoscale protrusions formed from such material. 


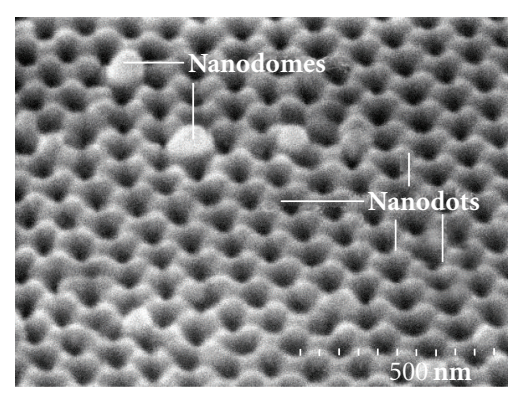

FIgURE 1: FE-SEM images of a $1 \times 1 \mu \mathrm{m}^{2}$ area of an anodic porous alumina film after PLD of LaS. The nanoprotrusions are the nanodomes and nanodots indicated in the figure.

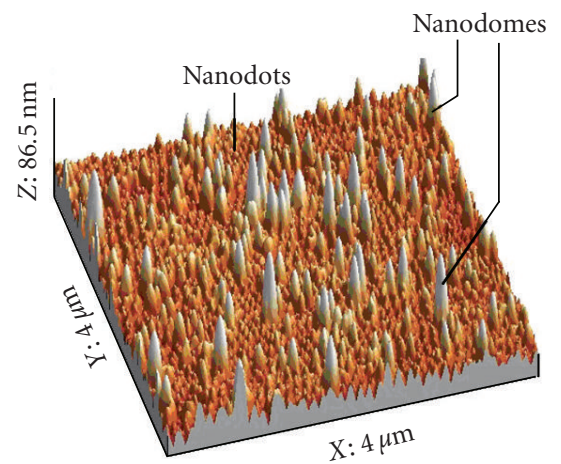

Figure 2: Three-dimensional AFM image of an area of $4 \times 4 \mu \mathrm{m}^{2}$ of the alumina template after PLD of LaS. The tallest irregular features are the LaS nanodomes. The smaller and more regular pyramidal structures are the LaS nanodots forming in the regions between adjacent pores.

In this letter, we report FE properties of arrays of LaS nanoprotrusions self-assembled with a density as high as $10^{9} / \mathrm{cm}^{2}$. These nanoprotrusions, called nanodomes and nanodots hereafter, are produced when LaS is pulse-laserdeposited on nanoporous anodic alumina films containing hexagonal arrays of cylindrical pores that are $\sim 50 \mathrm{~nm}$ wide and $\sim 500 \mathrm{~nm}$ deep. The details of the self-assembly growth of arrays of nanoprotrusions using a porous anodic alumina film as a template are given in [13].

The nanodomes are cone-shaped LaS structures with base diameter $2 r$ ranging from 50 to $100 \mathrm{~nm}$ and height $h$ ranging from 100 to $150 \mathrm{~nm}$. They were found to grow on the boundaries separating regions of the anodic alumina film that have near perfect pore ordering. Their density is $\sim 10^{9} / \mathrm{cm}^{2}$, as extracted from the field emission-scanning electron micrographs (FE-SEM) and atomic force micrographs (AFM) such as those shown in Figures 1 and 2, respectively. In addition to nanodomes, nanodots (or quantum dots) of LaS nucleate on top of the alumina islands between adjacent pores. These are sites of minimal strain. The dots have a diameter $2 r \sim 50 \mathrm{~nm}$, a density equal to the pore density $\left(10^{10} / \mathrm{cm}^{2}\right)$, and an aspect ratio $h / 2 r$ less than 1 .

The SAFEM technique was used to measure the FE current-voltage $(I-V)$ characteristics at different surface locations. As the nanowires are buried inside the pores, they contribute very little, if any at all, to the net FE current. Moreover, FE measurements from the arrays of nanodomes and nanodots were possible because the regions joining adjacent nanodomes and nanodots are covered with a thin percolating network of LaS over the entire array. Its presence was confirmed by measuring a low resistance value of about $2 \mathrm{Ohms}$ between two electrical contacts about $1 \mathrm{~cm}$ apart. For each location, a full set of $I-V$ characteristics (total measured current versus applied voltage) for different values of $d$, the distance between the cathode surface and the probe ball, was measured. This set of measurements was then analyzed in order to extract the apparent current density $\alpha J$ versus actual applied local field $\gamma F$, where $\gamma$ is the local geometrical field enhancement at the surface of the cathode [14], $F$ is the notional electric field at the corresponding flat LaS cathode surface (when $\gamma=1$ ), $\alpha$ a factor taking into account the actual emitting area, and $J$ the notional FE current density. In Figure 2, we see that the nanodome structures dominate the landscape and therefore they have the highest field enhancement factor $\gamma$. The relative contribution of the nanodots to the total FE current is minor due to a smaller value of the $\gamma$ associated with them. In all probability, the majority of the measured FE current is then extracted from the highest nanodomes.

In order to assess the FE properties from LaS nanoprotrusions, we have compared the FE from nanodome cathodes with that from planar thin film LaS cathodes. This comparative methodology was chosen because a precise quantitative estimation of $\gamma$ from topographic measurements is still subject to controversy, except for a flat surface. These experimental measurements were performed for both LaS thin films of $100 \mathrm{~nm}$ thickness on Si wafers and $\mathrm{LaS}$ nanodomes on anodic alumina films. The analysis consisted of the following steps.

(1) In order to restrict the analysis of the differences in the $I-V$ data to the surface morphology of the cathodes, both SAFEM measurements were performed with the same probe ball-to-cathode distance $d=3.65 \mu \mathrm{m}$. Typical $I-V$ characteristics are shown in Figure 3 for both thin film and nanodome cathodes. We found that in order to extract the same total FE current, the applied voltage for nanodome cathodes was $\sim 3.5$ times less than that for thin film cathodes. This indicates clearly the contribution of the protruding geometry of the nanodomes to the FE current.

(2) The total FE current versus applied voltage $(I-V)$ characteristics were measured for different values of the probe-ball-to-cathode-surface distance $d$. From these data, the apparent current densities $\left(\alpha_{t f} J\right.$ and $\left.\alpha_{n d} J\right)$ as a a function of the applied local field were extracted $[15,16]$, assuming that the applied local electric field included the local enhancement factor $\gamma$, that is, $\gamma_{t f} F$ and $\gamma_{n d} F$, for LaS thin film and nanodome cathodes, respectively. $\alpha_{t f}$ and $\alpha_{n d}$ are parameters taking into account the actual emitting areas from low work function nanocrystallite zones [12] and the density of emitting nanodomes. The corresponding Fowler-Nordheim plots $\left(\ln \left(\alpha J /(\gamma F)^{2}\right)\right.$ versus $\left.1 / \gamma F\right)$ of the data are shown in Figure 4. As a result of the field 


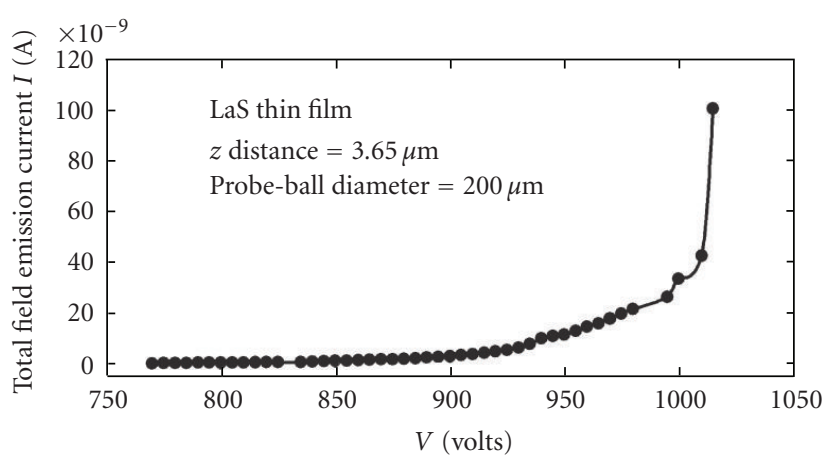

(a)

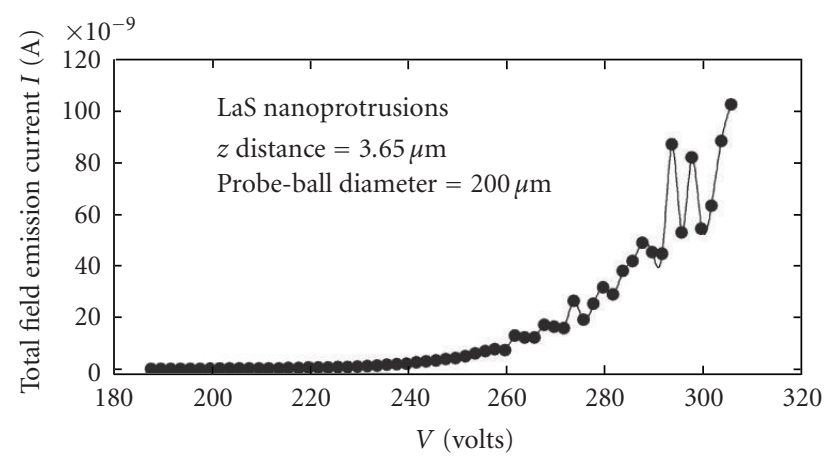

(b)

FIgure 3: (a) Typical $I$ - $V$ characteristic recorded using the SAFEM technique for FE from LaS thin film deposited via PLD on a Si substrate. (b) The same for LaS nanostructures self-assembled on a porous anodic alumina template. To allow immediate comparison, the sample to SAFEM probe distance is kept the same in both measurements.

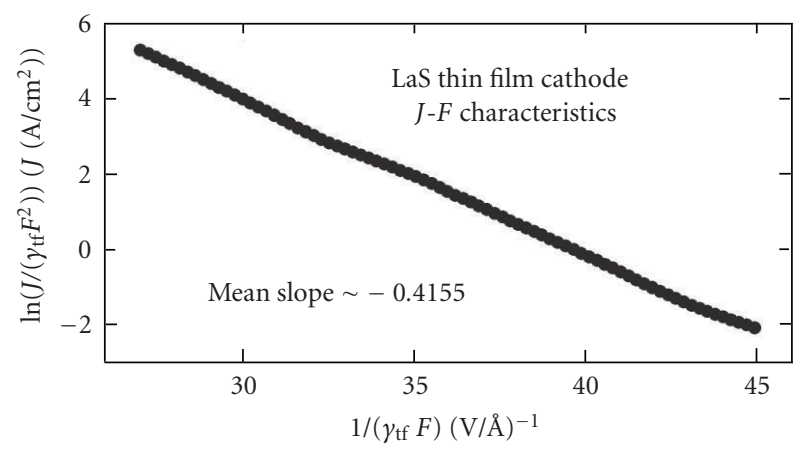

(a)

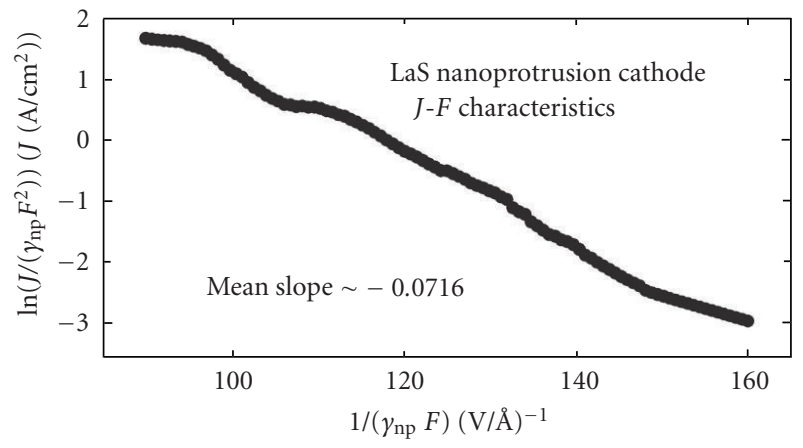

(b)

FIgURE 4: Fowler-Nordheim plots of the FE data corresponding to the cathodes shown in Figure 3.

enhancement factor, the emission area for nanodome cathodes was restricted to the nanodome apexes. The actual current densities $J$ extracted from each nanodome are then much more important than the values of the apparent current densities in the plot of Figure 4 . The exact estimation of the ratio $\alpha_{n d} / \alpha_{t f}$ would require a self-consistent approach, which is in progress, but one can estimate it in first approximation to be proportional to the inverse of the density of the emitting nanodomes. This ratio could be in the range of $10^{4}$ to $10^{5}$.

(3) The FE analysis for both cathodes, presented in the previous paragraph, was limited only to the region where the analytical FE relation developed by Murphy and Good is applicable $[15,16]$. Under such conditions, the slopes $m_{t f}$ and $m_{n d}$ of the Fowler-Nordheim plots are, respectively, proportional to $\Phi^{3 / 2} / \gamma_{t f}$ and $\Phi^{3 / 2} / \gamma_{n d}$, where $\Phi$ is the effective work function of the cathodes. In Figure 4, a straight line fit to the FowlerNordheim plots of the data gave slope values $m_{t f}=$ -0.4155 and $m_{n d}=-0.0716$ for $\mathrm{LaS} / \mathrm{Si}$ and nanodome cathodes, respectively.

(4) Under the assumption that the nanodome cathodes have the same effective work function as the planar cathodes on $\mathrm{Si}$, that is, $\Phi \sim 1 \mathrm{eV},[12]$ the ratio of the slopes extracted from the Fowler-Nordheim plots is equal to the inverse ratio of the field enhancement factors. Since AFM measurements of LaS thin films on Si substrates have shown that surface corrugation is negligible $[10,11]$, the error introduced by taking $\gamma_{t f}=1$ is minor. Under this assumption, $\gamma_{n d}=5.8$. This value is in very good agreement with the geometrical aspect ratio of the tallest nanodomes observed by AFM [13].

In conclusion, the SAFEM technique was used to demonstrate successful FE from LaS nanodomes grown by PLD on top of flexible alumina templates. The SAFEM measurements were found to be reproducible after a period of several months without taking any special precaution to store the template. LaS nanodome arrays are therefore very stable field emitters with a work function around $1 \mathrm{eV}$. Even though not discussed here, cross sectional FE-SEM images have shown that LaS nanowires grow inside the pores with a density of $10^{10} / \mathrm{cm}^{2}$. The nanowires have a diameter of $50 \mathrm{~nm}$ and a maximum length equal to the length of the pores $(\sim 500 \mathrm{~nm})$ [13]. This density could approach $10^{11} / \mathrm{cm}^{2}$ by using templates with pores size down to $10 \mathrm{~nm}$. Actually, the exposure of the tips of the LaS nanowires filling the pores can be done by etching the back aluminum substrate and the 
small alumina barrier at the bottom of the pores, which could lead to field enhancement factors for the LaS nanowires more comparable to those reported for CNTs. This would lead to a much lower threshold voltage for field emission compared to the one reported here and a large increase in the density of nanoemitters culminating in the realization, therefore the realization of flexible cold cathodes using a self-assembling process with reliable and low cost production.

\section{ACKNOWLEDGMENT}

The work at the University of Cincinnati and Virginia Commonwealth University is financially supported by the US National Science Foundation under the collaborative GOALI Grant ECS-0523966. Part of this work was performed by M. Cahay under the Air Force Summer Fellowship Program, Contract No. FA9550-07-C-0052.

\section{REFERENCES}

[1] A. G. Rinzler, J. H. Hafner, P. Nikolaev, et al., "Unraveling nanotubes: field emission from an atomic wire," Science, vol. 269, no. 5230, pp. 1550-1553, 1995.

[2] R. H. Baughman, A. A. Zakhidov, and W. A. de Heer, "Carbon nanotubes-the route toward applications," Science, vol. 297, no. 5582, pp. 787-792, 2002.

[3] D. Temple, "Recent progress in field emitter array development for high performance applications," Materials Science and Engineering R, vol. 24, no. 5, pp. 185-239, 1999.

[4] M. Mauger, V. T. Binh, A. Levesque, and D. Guillot, "Freestanding vertically aligned arrays of individual carbon nanotubes on metallic substrates for field emission cathodes," Applied Physics Letters, vol. 85, no. 2, pp. 305-307, 2004.

[5] Y. B. Li, Y. Bando, D. Golberg, and K. Kurashima, "Field emission from $\mathrm{MoO}_{3}$ nanobelts," Applied Physics Letters, vol. 81, no. 26, pp. 5048-5050, 2002.

[6] Y.-H. Lee, C.-H. Choi, Y.-T. Jang, et al., "Tungsten nanowires and their field electron emission properties," Applied Physics Letters, vol. 81, no. 4, pp. 745-747, 2002.

[7] J. Chen, S. Z. Deng, N. S. Xu, et al., "Field emission from crystalline copper sulphide nanowire arrays," Applied Physics Letters, vol. 80, no. 19, pp. 3620-3622, 2002.

[8] C. Y. Zhi, X. D. Bai, and E. G. Wang, "Synthesis and fieldelectron-emission behavior of aligned GaAs nanowires," Applied Physics Letters, vol. 86, no. 21, Article ID 213108, 3 pages, 2005.

[9] R. C. Wang, C. P. Liu, J. L. Huang, S.-J. Chen, Y.-K. Tseng, and S.-C. Kung, "ZnO nanopencils: efficient field emitters," Applied Physics Letters, vol. 87, no. 1, Article ID 013110, 3 pages, 2005.

[10] S. Fairchild, J. Jones, M. Cahay, et al., "Pulsed laser deposition of lanthanum monosulfide thin films on silicon substrates," Journal of Vacuum Science \& Technology B, vol. 23, no. 1, pp. 318-321, 2005.

[11] M. Cahay, K. Garre, X. Wu, D. Poitras, D. J. Lockwood, and S. Fairchild, "Physical properties of lanthanum monosulfide thin films grown on (100) silicon substrates," Journal of Applied Physics, vol. 99, no. 12, Article ID 123502, 6 pages, 2006.

[12] V. Semet, M. Cahay, V. T. Binh, S. Fairchild, X. Wu, and D. J. Lockwood, "Patchwork field emission properties of lan- thanum monosulfide thin films," Journal of Vacuum Science \& Technology B, vol. 24, no. 5, pp. 2412-2416, 2006.

[13] M. Cahay, K. Garre, J. W. Fraser, et al., unpublished.

[14] R. G. Forbes, C. J. Edgcombe, and U. Valdrè, "Some comments on models for field enhancement," Ultramicroscopy, vol. 95, pp. 57-65, 2003.

[15] V. Semet, R. Mouton, and V. T. Binh, "Scanning anode field emission microscopy analysis for studies of planar cathodes," Journal of Vacuum Science \& Technology B, vol. 23, no. 2, pp. 671-675, 2005.

[16] V. Semet, C. Adessi, and V. T. Binh, "Field emission from low barrier surface cathodes," in Proceedings of the 19th International Vacuum Nanoelectronics Conference, and the 50th International Field Emission Symposium (IVNC/IFES '06), pp. 2526, Guilin, China, July 2006. 

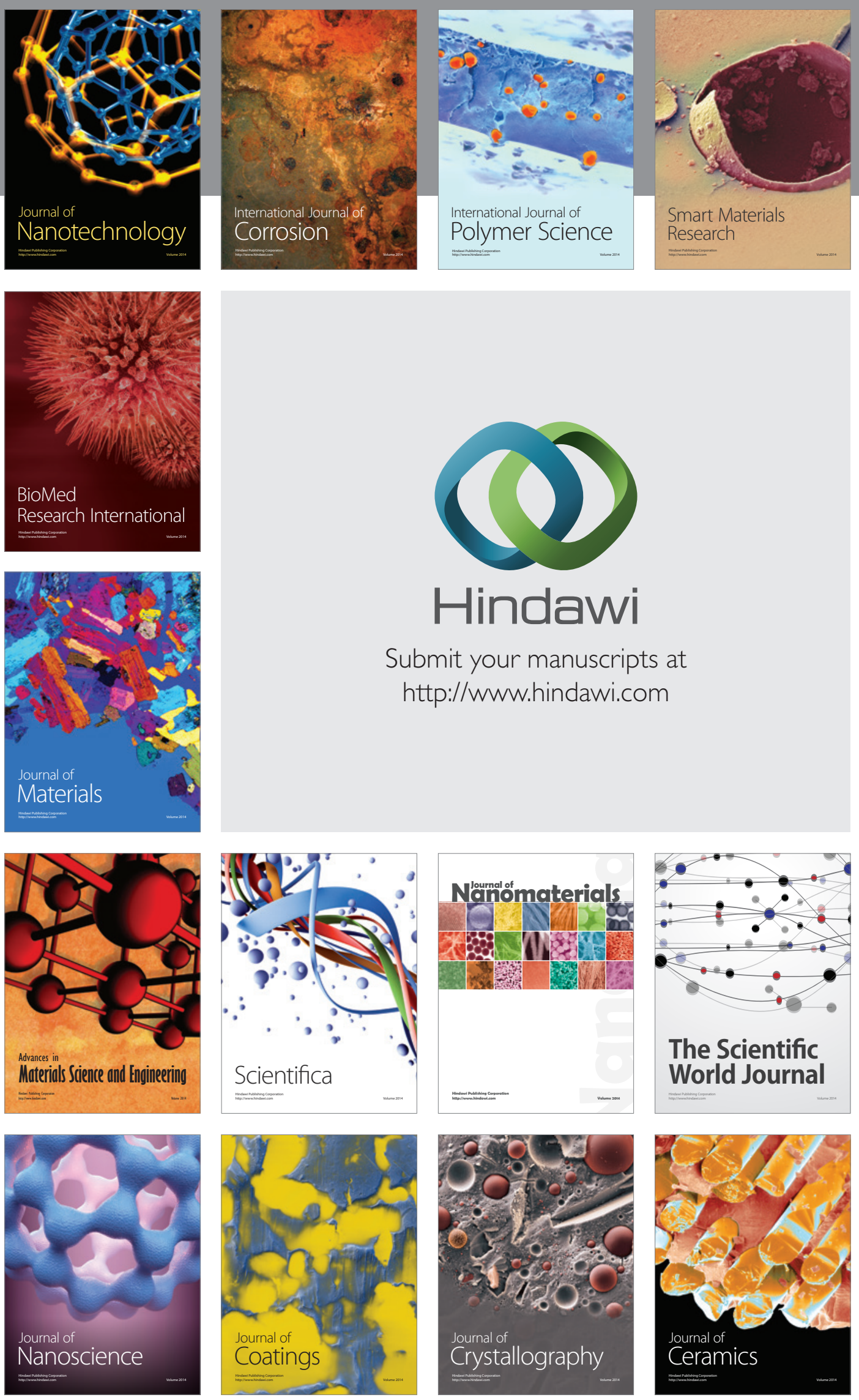

The Scientific World Journal

Submit your manuscripts at

http://www.hindawi.com

\section{World Journal}

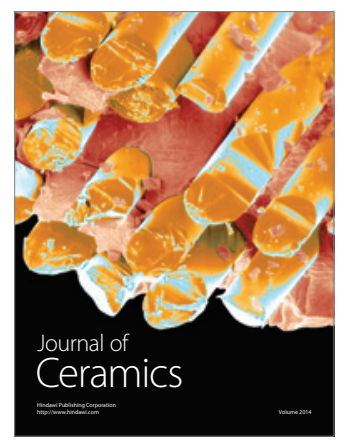

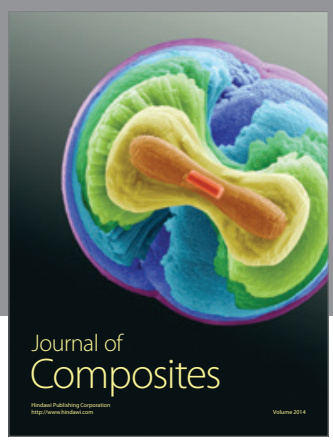
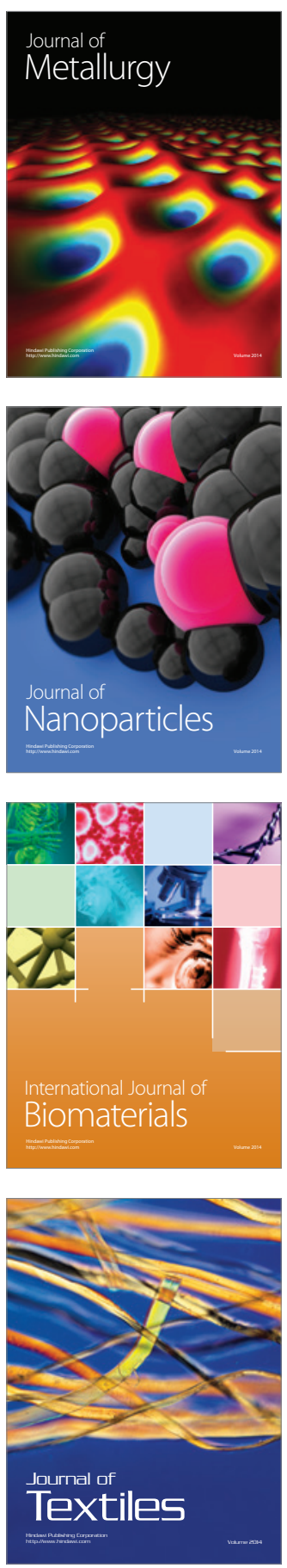